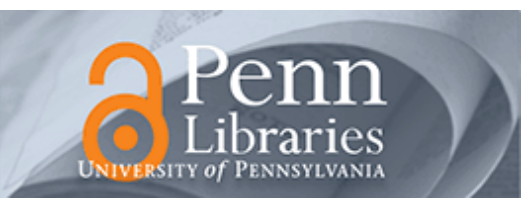

University of Pennsylvania

ScholarlyCommons

January 2004

\title{
Design and Implementation of Near-Field, Wideband Synthetic Aperture Beamformers
}

\author{
Fauzia Ahmad \\ Villanova University \\ Gordon J. Frazer \\ Defence Science and Technology Organisation \\ Saleem A. Kassam \\ University of Pennsylvania, kassam@seas.upenn.edu \\ Moeness G. Amin \\ Villanova University
}

Follow this and additional works at: https://repository.upenn.edu/ese_papers

\section{Recommended Citation}

Fauzia Ahmad, Gordon J. Frazer, Saleem A. Kassam, and Moeness G. Amin, "Design and Implementation of Near-Field, Wideband Synthetic Aperture Beamformers", . January 2004.

Copyright 2004 IEEE. Reprinted from IEEE Transactions on Aerospace and Electronic Systems, Volume 40, Issue 1, January 2004, pages 206-220.

Publisher URL: http://ieeexplore.ieee.org/xpl/tocresult.jsp?isNumber=28783\&page=1

This material is posted here with permission of the IEEE. Such permission of the IEEE does not in any way imply IEEE endorsement of any of the University of Pennsylvania's products or services. Internal or personal use of this material is permitted. However, permission to reprint/republish this material for advertising or promotional purposes or for creating new collective works for resale or redistribution must be obtained from the IEEE by writing to pubs-permissions@ieee.org. By choosing to view this document, you agree to all provisions of the copyright laws protecting it.

This paper is posted at ScholarlyCommons. https://repository.upenn.edu/ese_papers/37

For more information, please contact repository@pobox.upenn.edu. 


\title{
Design and Implementation of Near-Field, Wideband Synthetic Aperture Beamformers
}

\author{
Abstract \\ A coarray-based near-field, wideband synthetic aperture beamformer using stepped-frequency signal \\ synthesis and post-data acquisition processing is presented. While coarray techniques offer significant \\ reduction in the number of array elements for a given angular resolution, the hybrid subarray-stepped \\ frequency realization of wideband systems simplifies implementations and offers flexibility in \\ beamforming. Proof of concept is provided using real data collected in an anechoic chamber for several \\ pulse shapes and array weightings.

\section{Comments} \\ Copyright 2004 IEEE. Reprinted from IEEE Transactions on Aerospace and Electronic Systems, Volume 40, \\ Issue 1, January 2004, pages 206-220. \\ Publisher URL: http://ieeexplore.iee.. rg/xpl/tocresult.jsp?isNumber=28783\&page=1 \\ This material is posted here with permission of the IEEE. Such permission of the IEEE does not in any way \\ imply IEEE endorsement of any of the University of Pennsylvania's products or services. Internal or \\ personal use of this material is permitted. However, permission to reprint/republish this material for \\ advertising or promotional purposes or for creating new collective works for resale or redistribution must \\ be obtained from the IEEE by writing to pubs-permissions@ieee.org. By choosing to view this document, \\ you agree to all provisions of the copyright laws protecting it.
}




\section{Design and Implementation of Near-Field, Wideband Synthetic Aperture Beamformers}

\section{FAUZIA AHMAD}

Villanova University

\section{GORDON J. FRAZER}

Defence Science and Technology Organisation

Australia

SALEEM A. KASSAM, Fellow, IEEE

University of Pennsylvania

MOENESS G. AMIN, Fellow, IEEE

Villanova University

A coarray-based near-field, wideband synthetic aperture beamformer using stepped-frequency signal synthesis and post-data acquisition processing is presented. While coarray techniques offer significant reduction in the number of array elements for a given angular resolution, the hybrid subarray-stepped frequency realization of wideband systems simplifies implementations and offers flexibility in beamforming. Proof of concept is provided using real data collected in an anechoic chamber for several pulse shapes and array weightings.

Manuscript received January 6, 2003; revised June 19, 2003; released for publication September 10, 2003.

IEEE Log No. T-AES/40/1/826467.

Refereeing of this contribution was handled by S. S. Toumodge.

This work was supported by DARPA under Grant MDA972-02-1-0022 and in part by ONR Grant N00014-98-1-0176. The content of the information does not necessarily reflect the position or the policy of the Government, and no official endorsement should be inferred.

Authors' addresses: F. Ahmad and M. G. Amin, Center for Advanced Communications, Villanova University, 800 Lancaster Ave., Villanova, PA 19085, E-mail: (fahmad@ece.vill.edu); G. J. Frazer, ISR Division, Defence Science and Technology Organisation, PO Box 1500, Edinburgh, SA, 5111 Australia; S. A. Kassam, Dept. of Electrical and Systems Engineering, University of Pennsylvania, 200 S. 33rd St., Philadelphia, PA 19104.

0018-9251/04/\$17.00 (c) 2004 IEEE

\section{INTRODUCTION}

Spatial arrays of sensor elements deployed over a plane region are often used to image sources of reflected power in radar, sonar, and ultrasound applications. The array of sensors samples the reflected field at a fixed set of locations. The sensor data are then processed using signal processing techniques such as beamforming to produce an image characterizing the distribution of the objects in the field of view of the active array imaging system. The scene to be imaged is often illuminated with short duration pulses to achieve good range resolution.

The concept of coarrays $[1,2]$ facilitates addressing fundamental array signal processing issues in a simplified context. The coarray was originally defined for narrowband far-field active imaging [1], and is represented by a set of pair-wise sums of the position vectors of the elements in the transmit and receive apertures. The concept of coarrays was extended to wideband imaging in [3], where it was shown that wideband operation adds extra points at specific locations in the sum coarray. This property has been utilized to enhance the angular resolution achievable with a limited number of array elements [4].

In addition to coarrays, subarray processing is also pertinent to the proposed imaging technique. An aperture synthesis technique using subarrays, was first proposed in [5] for ultrasound applications. In this scheme, the transmit and receive arrays are divided into subarray pairs, where each subarray consists of a single transmitter and one or more receivers. The subarrays are used independently to form component complex images of the scene by post-data acquisition beamforming [5]. These independent component images are then added coherently to obtain the composite complex amplitude image with the desired spatial resolution. This scheme was later generalized in $[6,7]$ to incorporate subarrays composed of multiple transmitters using the concept of sum coarray, where it was shown that the composite image, obtained using the subarray aperture synthesis scheme, has an effective sum coarray that is the union of the individual coarrays corresponding to each transmit/receive subarray pair.

Until recently, the subarray aperture synthesis scheme was primarily used in ultrasound imaging. It was recently extended to microwave imaging, particularly for through-the-wall operation, by us in [8], and by Benjamin, et al. in [9]. Both these systems use wideband pulses for imaging, and divide the transmit and receive arrays into single transmitter/single receiver subarray pairs. All the elements of the intended transmit and receive arrays have to be physically present, and share a pair of processing channels via a subarray multiplexer. 
Although the work in [9] uses single transmitter/single receiver pairs, our work in [8] provides a general framework for array synthesis, and permits the realization of desired imaging characteristics by use of the synthesized aperture. More specifically, any arbitrary desired coarray weighting can be placed on the entire sum coarray by imparting appropriate weights to the component sum coarrays, corresponding to each subarray pair. This can be used to acheive greater control over the sidelobe structure of the point spread function (PSF) [6]. It is important to note that the aperture synthesis scheme does not readily suggest itself without considering the sum coarrays.

We present a new imaging system based on the concepts of subarrays, coarrays, and stepped-frequency signal synthesis. The imaging system described herein makes use of an alternative implementation of the aperture synthesis scheme in which a single subarray, consisting of one transmitter and one receiver, is used. The full transmit and receive array is realized by moving this subarray to different locations forming the array aperture. This reduces the cost of the system since only two antenna elements, and a pair of transmit and receive processing apparatus is required. The resolution of the larger array can thus be obtained with significant reduction in cost at the expense of increased data acquisition time. Instead of using pulses, we synthesize a wideband signal using the stepped-frequency approach. The synthesized signal along with post-data acquisition processing is used to implement a near-field synthetic aperture beamformer in the frequency domain. It is shown that the application of the stepped-frequency approach leads to a flexible and simplified implementation of the transmit and receive beamformers.

The paper is organized as follows. The concept of the sum coarrays is explained in Section II. In Section III, we describe the post-data acquisition processing and introduce the stepped-frequency synthesis of the wideband signal. The frequency domain implementation of the near-field beamformer is also discussed. Section IV describes the system parameters chosen for the implementation of the proposed beamformer in an anechoic chamber. The performance of the proposed system using real data, demonstrating the working of the wideband, near-field beamformer in a microwave imaging scenario, is shown in Section V. Section VI contains the concluding remarks.

\section{SUM COARRAY}

For narrowband far-field active imaging, the sum coarray is defined to be the set [1]

$$
C_{S}=\left\{\mathbf{z}: \mathbf{z}=\mathbf{x}+\mathbf{y}, \mathbf{x} \in S_{T}, \mathbf{y} \in S_{R}\right\}
$$

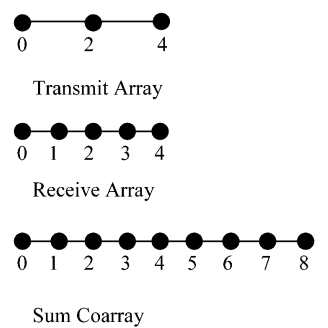

Fig. 1. Transmit and receive arrays and the corresponding sum coarray.

where $S_{T}$ and $S_{R}$ are the sets of element position vectors in the transmit and receive apertures, respectively. An example illustrating the sum coarray is shown in Fig. 1. The figure shows an active imaging system, which uses a 3-element transmit and a 5-element receive line array for transmission and reception, and its corresponding sum coarray. For this system, the maximum number of points that the sum coarray can have is 15 . However, since every pair of transmit and receive elements does not contribute to the formation of only one coarray point, the actual number of points in the sum coarray is 9 .

To understand the importance of the sum coarray, let us focus our attention on linear imaging techniques for narrowband imaging of far-field scenes. In "linear imaging," the image is the convolution of the PSF with the true reflectivity distribution of the scene. The PSF, which determines the basic characteristic of the imaging system, is simply the response of the system to a point source. For weighted beamforming, the PSF is the Fourier transform of a weighting function that has support on the sum coarray. The coarray weighting function, imposed by beamforming using single snapshot, is the convolution of the transmit and receiver array apodizations. In fact, it is only by way of modification of the PSF that the characteristics of the linearly formed image can be influenced. Therefore, the sum coarray determines the class of PSFs that an active imaging system can achieve.

The explicit relationship between the PSF $P(\mathbf{u})$ and the sum coarray weighting $\gamma(\mathbf{z})$ is

$$
P(\mathbf{u})=\int \gamma(\mathbf{z}) \exp \left(j 2 \pi \mathbf{u} \cdot \frac{\mathbf{z}}{\lambda}\right) d \mathbf{z}
$$

where $\lambda$ is the wavelength of operation and $\mathbf{u}=$ $(\sin \theta \cos \phi, \sin \theta \sin \phi$ ) is the reduced angular coordinate. We note that more precisely, $P(\mathbf{u})$ is the Fourier transform of the weighting function $\lambda \gamma(\lambda \mathbf{z})$. Thus, a more explicit definition of the sum coarray would dilate it by the factor $1 / \lambda$, i.e., if $C_{S}$ is the sum coarray of a given array, then the dilated sum coarray, denoted by $\lambda \cdot C_{S}$ is

$$
\lambda \cdot C_{S}=\left\{\mathbf{z} / \lambda: \mathbf{z} \in C_{S}\right\} .
$$

This dilated coarray comes into play when we image the scene using wideband signals. In fact, for 


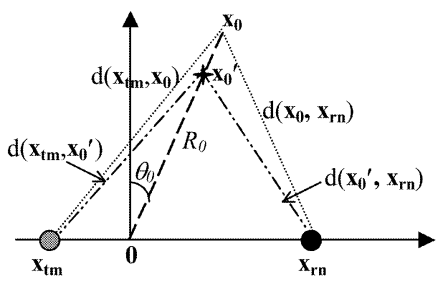

Fig. 2. Moving target scenario.

wideband operation, the sum coarray is the union of the dilated sum coarrays corresponding to all the frequencies constituting the bandwidth of the transmitted signal [3], i.e.

$$
C_{\mathrm{WB}}=\bigcup_{\lambda} \lambda \cdot C_{S} .
$$

Note that multi-frequency operation adds extra points at specific locations in the original $C_{S}$. This richer coarray can be used to enhance the angular resolution achievable with a limited number of array elements.

\section{DESIGN OF NEAR-FIELD WIDEBAND SYNTHETIC APERTURE BEAMFORMER}

In this section, we describe in detail our approach to the design of the near-field, wideband synthetic aperture beamformer in terms of both the post-data acquisition processing for aperture synthesis and the stepped-frequency synthesis of the wideband signal. This design is applicable to both active line and planar arrays in which the transmit and receiver elements are either collocated or are at physically separate locations. Since we use separate transmit and receive line arrays in the implementation of the proposed beamformer, we limit our discussion to such systems.

\section{A. System Model}

Consider a single transmitter and a single receiver subarray, which are used to synthesize an $M$-element transmit and an $\mathrm{N}$-element receive line array, both located along the $\mathrm{x}$ axis. The region to be imaged is located in the $\mathrm{x}-\mathrm{z}$ plane, along the positive $\mathrm{z}$ axis. Let the transmitter, placed at the $m$ th transmit location, illuminate the scene with a wideband signal $s(t)$. The reflection by any target located in the region being imaged is measured and stored by the receiver at the $n$th receive location. Consider a single point target moving towards the origin with a velocity $v_{0}$ as shown in Fig. 2. Let the target position be $\mathbf{x}_{0}=\left(x_{0}, z_{0}\right)$ (or in polar coordinates, $\left.\mathbf{x}_{0}=\left(R_{0}, \theta_{0}\right)\right)$ at reference time $t=0$. The target position at any time $t$ is given by [10-12]

$$
\mathbf{x}_{0}^{\prime}=\left(x_{0}^{\prime}, z_{0}^{\prime}\right)=\left(R_{0}-v_{0} t, \theta_{0}\right) .
$$

Then, the signal received by the $n$th receiver is given by $s\left(t-\left(d\left(\mathbf{x}_{\mathbf{t m}}, \mathbf{x}_{0}^{\prime}\right)+d\left(\mathbf{x}_{0}^{\prime}, \mathbf{x}_{\mathbf{r n}}\right)\right) / c\right)$, where $d(\cdot, \cdot)$ is the Cartesian distance between two position vectors, $\mathbf{x}_{\mathbf{t m}}$ and $\mathbf{x}_{\mathbf{r n}}$ are the positions vectors of the $m$ th transmit and $n$th receive locations, respectively, and $c$ is the velocity of light.

From Fig. 2, and using simple trigonometry relations, it is clear that

$$
\begin{aligned}
d\left(\mathbf{x}_{\mathbf{t m}}, \mathbf{x}_{0}^{\prime}\right)+d\left(\mathbf{x}_{0}^{\prime}, \mathbf{x}_{\mathbf{r n}}\right) \\
=\sqrt{\left(R_{0}-v_{0} t\right)^{2}+x_{t m}^{2}-2 x_{t m}\left(R_{0}-v_{0} t\right) \sin \theta_{0}} \\
\quad+\sqrt{\left(R_{0}-v_{0} t\right)^{2}+x_{r n}^{2}-2 x_{r n}\left(R_{0}-v_{0} t\right) \sin \theta_{0}} \\
=\sqrt{R_{0}^{2}+x_{t m}^{2}-2 R_{0} x_{t m} \sin \theta_{0}+v_{0}^{2} t^{2}-2 v_{0} t\left(R_{0}-x_{t m} \sin \theta_{0}\right)} \\
\quad+\sqrt{R_{0}^{2}+x_{r n}^{2}-2 R_{0} x_{r n} \sin \theta_{0}+v_{0}^{2} t^{2}-2 v_{0} t\left(R_{0}-x_{r n} \sin \theta_{0}\right)} .
\end{aligned}
$$

Since

$$
\begin{aligned}
& d\left(\mathbf{x}_{\mathbf{t m}}, \mathbf{x}_{0}\right)=\sqrt{R_{0}^{2}+x_{t m}^{2}-2 R_{0} x_{t m} \sin \theta_{0}} \\
& d\left(\mathbf{x}_{0}, \mathbf{x}_{r n}\right)=\sqrt{R_{0}^{2}+x_{r n}^{2}-2 R_{0} x_{r n} \sin \theta_{0}} .
\end{aligned}
$$

Therefore, (5) can be written as

$$
\begin{aligned}
d\left(\mathbf{x}_{\mathbf{t m}}, \mathbf{x}_{0}^{\prime}\right)+d\left(\mathbf{x}_{0}^{\prime}, \mathbf{x}_{\mathbf{r n}}\right) & \\
= & d\left(\mathbf{x}_{\mathbf{t m}}, \mathbf{x}_{0}\right) \sqrt{1+\frac{\left(v_{0}^{2} t^{2}-2 v_{0} t\left(R_{0}-x_{t m} \sin \theta_{0}\right)\right.}{d^{2}\left(\mathbf{x}_{\mathbf{t m}}, \mathbf{x}_{0}\right)}} \\
& +d\left(\mathbf{x}_{0}, \mathbf{x}_{\mathbf{r n}}\right) \sqrt{1+\frac{\left(v_{0}^{2} t^{2}-2 v_{0} t\left(R_{0}-x_{r n} \sin \theta_{0}\right)\right.}{d^{2}\left(\mathbf{x}_{0}, \mathbf{x}_{\mathbf{r n}}\right)}}
\end{aligned}
$$

Using the binomial theorem, (6) can be rewritten as

$$
\begin{aligned}
d\left(\mathbf{x}_{\mathbf{t m}}, \mathbf{x}_{0}^{\prime}\right)+d\left(\mathbf{x}_{0}^{\prime}, \mathbf{x}_{\mathbf{r n}}\right) \\
=d\left(\mathbf{x}_{\mathbf{t m}}, \mathbf{x}_{0}\right)+d\left(\mathbf{x}_{0}, \mathbf{x}_{\mathbf{r n}}\right) \\
-\left[\frac{v_{0} t\left(R_{0}-x_{t m} \sin \theta_{0}\right)}{d\left(\mathbf{x}_{\mathbf{t m}}, \mathbf{x}_{0}\right)}+\frac{v_{0} t\left(R_{0}-x_{r n} \sin \theta_{0}\right)}{d\left(\mathbf{x}_{0}, \mathbf{x}_{\mathbf{r n}}\right)}\right] \\
\quad+\text { higher order terms. }
\end{aligned}
$$

Equation (7) provides the general Cartesian distance expression, incorporating target movement and time dependency of the scene. For the proposed aperture synthesis scheme to work, the scene being imaged should not, however, change during the observation period using the entire transmit and receive array locations for imaging. In through-the-wall microwave imaging applications [13-18], the velocities of the targets involved are small and satisfy this condition. Hence, moving targets will be treated as stationary targets during the observation period, and we can ignore the terms involving $v_{0}$ and its powers in (7). However, note that moving target scenarios may still 


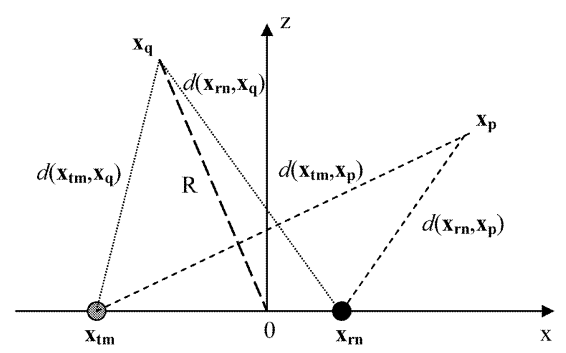

Fig. 3. Geometry of scene being imaged.

be imaged by extracting Doppler from a repeated sequence of coherent measurements.

\section{B. Post-data Acquisition Processing}

For the case of a single point target located at $\mathbf{x}_{\mathbf{p}}=\left(x_{p}, z_{p}\right)$ in the region being imaged, the output of the $n$th receiver is given by $a\left(\mathbf{x}_{\mathbf{p}}\right) s\left(t-\tau_{m n}\right)$. Here $a\left(\mathbf{x}_{\mathbf{p}}\right)$ is the complex reflectivity of the point target, and $\tau_{m n}$ is the propagation delay encountered by the signal as it travels from the $m$ th transmitter to the target located at $\mathbf{x}_{\mathbf{p}}$, and back to the $n$th receiver, as shown in Fig. 3, and is given by

$$
\tau_{m n}=\frac{d\left(\mathbf{x}_{\mathbf{t m}}, \mathbf{x}_{\mathbf{p}}\right)}{c}+\frac{d\left(\mathbf{x}_{\mathbf{p}}, \mathbf{x}_{\mathbf{r n}}\right)}{c} .
$$

This process is repeated, with the transmitter at the $m$ th location, until all the $N$ receive locations have been used sequentially. The $N$ outputs, thus obtained, are processed as follows.

The region of interest is divided into a finite number of pixels in range and angle. The complex composite signal corresponding to the image of the $q$ th pixel, located at $\mathbf{x}_{\mathbf{q}}$ (at range $R$ in the direction $\theta$ ), is obtained by applying time delays and weights to the outputs of the $N$ receivers, and summing them. The resulting output for a single target case is given by

$$
z_{m}(t)=\sum_{n=1}^{N} w_{r n} a\left(\mathbf{x}_{\mathbf{p}}\right) s\left(t-\tau_{m n}-\tilde{\tau}_{m n}\right)
$$

where $w_{r n}$ is the weight applied to the output of the $n$th receiver, and $\tilde{\tau}_{m n}$ is the focusing delay applied to the output of the $n$th receiver when the transmitter is at the $m$ th location, and is given by (see Fig. 3)

$$
\tilde{\tau}_{m n}=\frac{2 R}{c}-\frac{d\left(\mathbf{x}_{\mathbf{t m}}, \mathbf{x}_{\mathbf{q}}\right)}{c}-\frac{d\left(\mathbf{x}_{\mathbf{q}}, \mathbf{x}_{\mathbf{r n}}\right)}{c} .
$$

Note that if the target is indeed present at the $q$ th pixel location, i.e., $\mathbf{x}_{\mathbf{p}}=\mathbf{x}_{\mathbf{q}}$, then

$$
\tau_{m n}+\tilde{\tau}_{m n}=\frac{2 R}{c} \quad \text { for } n=1,2, \ldots, N .
$$

This means that all the outputs line up and add together to produce a coherently combined output. However, if no reflected radiation is picked up from the $q$ th pixel, then the same time delays will cause the receive elements' outputs to stagger in time, thereby producing a reduced combined output.

This process is repeated by sequential use of the $M$ transmit locations, one by one, and $M$ complex composite signals corresponding to the image of the $q$ th pixel are obtained. The final complex signal corresponding to the $q$ th pixel is obtained by the coherent weighted linear combination

$$
z(t)=\sum_{m=1}^{M} w_{t m} z_{m}(t)=\sum_{m=1}^{M} \sum_{n=1}^{N} w_{t m} w_{r n} a\left(\mathbf{x}_{\mathbf{p}}\right) s\left(t-\tau_{m n}-\tilde{\tau}_{m n}\right)
$$

where $w_{t m}$ is the weight applied to the component signal $z_{m}(t)$ obtained using the $m$ th transmitter. The complex amplitude image value for the $q$ th pixel is obtained from (11) as

$$
I\left(\mathbf{x}_{\mathbf{q}}\right)=\left.z(t)\right|_{t=2 R / c}
$$

where the sampling time $2 R / c$ corresponds to the time of flight of the wideband waveform for the focused range $R$.

This process is performed for all pixels in the region of interest to generate the composite image of the scene being imaged. The general case of multiple targets can be obtained by superposition.

Note that at no time is a formed beam transmitted. Rather, the effect of a focused transmit beam is created by a post-data acquisition processing. Thus, the data acquisition time is only a function of the number of transmitters and receivers, and the depth of view within the region of interest. This time does not depend on the number of pixels to be displayed in the final complex amplitude image. That is because electromagnetic energy is being projected into the entire region of interest at the same time, rather than point-by-point, in sequence. Also, the problems associated with the implementation of a scanning, focused transmitting beam are completely eliminated.

The antenna elements could have been used to synthesize planar arrays. With planar arrays, it would be possible to provide the desired continuous range focus in both azimuth and elevation.

\section{Stepped-Frequency Synthesis}

Consider the Fourier transform $S(\omega)$ of the transmitted wideband signal $s(t)$, given by

$$
s(t)=\frac{1}{2 \pi} \int_{-\infty}^{\infty} S(\omega) e^{j \omega t} d \omega .
$$

Using the time-shift property of the Fourier transform, we get

$$
s\left(t-\tau_{m n}-\tilde{\tau}_{m n}\right)=\frac{1}{2 \pi} \int_{-\infty}^{\infty} S(\omega) e^{j \omega t} \exp \left(-j \omega\left(\tau_{m n}+\tilde{\tau}_{m n}\right)\right) d \omega .
$$


Therefore, the frequency-domain representation of the underlying problem is obtained by using (11), (12), and (13),

$$
\begin{aligned}
I\left(\mathbf{x}_{\mathbf{q}}\right)= & \frac{1}{2 \pi} \int_{-\infty}^{\infty} S(\omega) e^{j \omega t} a\left(\mathbf{x}_{\mathbf{p}}\right) \sum_{m=1}^{M} \sum_{n=1}^{N} w_{t m} w_{r n} \\
& \times\left.\exp \left(-j \omega\left(\tau_{m n}+\tilde{\tau}_{m n}\right)\right) d \omega\right|_{t=2 R / c} .
\end{aligned}
$$

If $s(t)$ had been a narrowband signal of frequency $\omega_{c}$, i.e., $S(\omega)=P(\omega) \delta\left(\omega-\omega_{c}\right)$, expression (14) would take the form

$$
\begin{aligned}
I\left(\mathbf{x}_{\mathbf{q}}\right)= & \frac{1}{2 \pi} \int_{-\infty}^{\infty} P(\omega) e^{j \omega t} \delta\left(\omega-\omega_{c}\right) a\left(\mathbf{x}_{\mathbf{p}}\right) \sum_{m=1}^{M} \sum_{n=1}^{N} w_{t m} w_{r n} \\
& \times\left.\exp \left(-j \omega\left(\tau_{m n}+\tilde{\tau}_{m n}\right)\right) d \omega\right|_{t=2 R / c} \\
= & \frac{1}{2 \pi} P\left(\omega_{c}\right) e^{j \omega_{c} t} a\left(\mathbf{x}_{\mathbf{p}}\right) \sum_{m=1}^{M} \sum_{n=1}^{N} w_{t m} w_{r n} \\
& \times\left.\exp \left(-j \omega_{c}\left(\tau_{m n}+\tilde{\tau}_{m n}\right)\right)\right|_{t=2 R / c} .
\end{aligned}
$$

That is, the time delay $\tau_{m n}+\tilde{\tau}_{m n}$ would appear as a pure phase delay of the transmitted signal. For the wideband case, the expression in (14) is structurally the same as that for the narrowband case. However, the time delay $\tau_{m n}+\tilde{\tau}_{m n}$ now appears as a frequency-dependent phase delay [19]. This implies that beamforming can be achieved by transmitting monochromatic signals corresponding to the continuum of frequencies constituting the wideband signal spectrum $S(\omega)$, measuring the complex amplitude of the returns, performing synthetic focusing in the frequency domain for each frequency using a phase delay corresponding to that frequency, and then combining the results per (14).

A stepped-frequency approximation to the above approach would use a finite number $K$ of monochromatic signals with equi-spaced frequencies $\omega_{k}$ covering the desired bandwidth $\omega_{K-1}-\omega_{0}$

$$
\begin{aligned}
& \omega_{k}=\omega_{0}+k \Delta \omega, \quad \Delta \omega=\frac{\omega_{K-1}-\omega_{0}}{K-1} \\
& \text { for } k=0,1, \ldots, K-1 .
\end{aligned}
$$

Here, $\Delta \omega$ is the frequency step size.

Equation (14) can now be rewritten as

$$
\begin{aligned}
I\left(\mathbf{x}_{\mathbf{q}}\right)= & \frac{1}{2 \pi} \sum_{k=0}^{K-1} S\left(\omega_{k}\right) \exp \left(j \omega_{k} t\right) a\left(\mathbf{x}_{\mathbf{p}}\right) \sum_{m=1}^{M} \sum_{n=1}^{N} w_{t m} w_{r n} \\
& \times\left.\exp \left(-j \omega_{k}\left(\tau_{m n}+\tilde{\tau}_{m n}\right)\right)\right|_{t=2 R / c} .
\end{aligned}
$$

This expression forms the basis of the steppedfrequency implementation of the aperture synthesis scheme using subarrays.
Since we transmit and receive $K$ monochromatic signals for each subarray pair location, a total of $K M N$ transmissions and corresponding receptions of reflections need to be collected before the complete image can be obtained. On the other hand, for a pulsed waveform, just $M N$ measurements would suffice. This scheme is, therefore, more time consuming. However, it is cost-effective since we do not need expensive equipment for pulse generation and processing. Also, the proposed scheme offers implementation flexibility in the context of waveform design. With just these $K M N$ measurements, it is possible to form images of the scene by synthesizing a variety of wideband waveforms using appropriate spectral values $S\left(\omega_{k}\right)$ in (15). These images could be used for relative evaluations among prospective signals for a particular imaging application, as shown later. Alternatively, an optimized waveform could be designed by using (15) to achieve desired characteristics of the angular array response.

1) Interpretation of the Stepped-Frequency Signal: Since $S(\omega)$ is considered at uniform frequency samples spaced by $\Delta \omega$, the equivalent time signal is given by [20]

$$
\tilde{s}(t)=\frac{1}{\Delta \omega} \sum_{l=-\infty}^{\infty} s\left(t-\frac{2 \pi}{\Delta \omega} l\right) .
$$

The signal $\tilde{s}(t)$ consists of periodic replicas of the wideband signal $s(t)$ spaced at integer multiples of $2 \pi / \Delta \omega$. According to the Nyquist sampling theorem, if the wideband signal $s(t)$ is time-limited with

$$
s(t)=0 \quad|t|>T / 2
$$

then $\tilde{s}(t)$ will consist of non-overlapping replicas of $s(t)$ as long as

$$
\frac{2 \pi}{\Delta \omega} \geq T .
$$

If, however, this inequality is not satisfied, the replicas of $s(t)$ will overlap resulting in time-domain aliasing.

For practical purposes, the wideband pulse used for transmission is time-limited. This implies that the pulse spectrum will not be band-limited. A filtering operation can limit the effective spectral spread. An appropriate choice of both the pulse shape and the frequency response of the processing filter would result in a wideband pulse that has the desired bandwidth and is approximately time-limited, i.e., it satisfies the condition

$$
s(t) \approx 0 \quad|t|>T^{\prime} / 2 .
$$

Time-aliasing can be significantly reduced by selecting $\Delta \omega$ so that

$$
\frac{2 \pi}{\Delta \omega} \geq T^{\prime} .
$$

2) Practical Implementation of the SteppedFrequency Signal: The stepped-frequency signal 


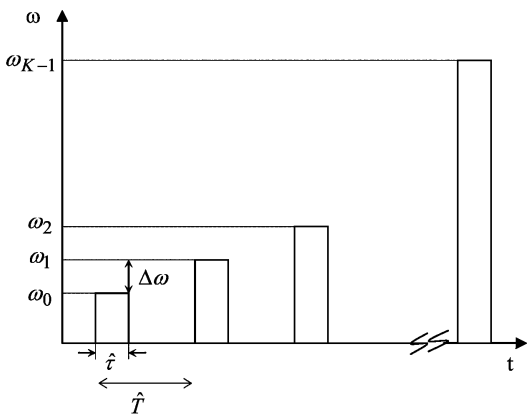

Fig. 4. Single burst of stepped-frequency signal.

can be generated as follows [10]. A series of $K$ narrowband pulses are transmitted, whose frequency is incremented by a fixed step of size $\Delta \omega$, as shown in Fig. 4. $\hat{T}$ is the narrowband pulse repetition interval and $\hat{\tau}$ is the narrowband pulsewidth. Each group of $K$ narrowband pulses is referred to as a burst.

The value of $\hat{T}$ can be determined from the range ambiguity associated with the stepped-frequency signal. It has been shown in [10] that by considering the phase difference of two received narrowband signals, which are separated in frequency by $\Delta \omega$, the maximum unambiguous range $R_{u}$ is given by

$$
R_{u}=\frac{c \pi}{\Delta \omega} .
$$

Therefore, the upper limit on the pulse repetition frequency is set by the maximum unambiguous range as $c / 2 R_{u}=\Delta \omega / 2 \pi$. This implies that the pulse repetition interval $\hat{T}$

$$
\hat{T} \geq \frac{2 \pi}{\Delta \omega} .
$$

This allows the backscattered signals from the farthest range of interest to reach the receiver before the signal source switches to the next frequency.

For example, for a stepped-frequency signal of $1 \mathrm{GHz}$ bandwidth centered at $2 \mathrm{GHz}$, with 801 steps of size $1.25 \mathrm{MHz}$, the pulse repetition frequency should be less than or equal to $1.25 \mathrm{MHz}$. Therefore, $\hat{T}$ should be greater than or equal to $0.8 \mu \mathrm{s}$.

The upper limit for $\hat{T}$ will be set by the target detectability requirement and that relates to the total energy of the burst consisting of $K$ narrowband pulses.

Note that for the proposed scheme, since a single transmitter and a single receiver subarray is used, $M N$ stepped-frequency bursts are required for data acquisition.

\section{SYSTEM PARAMETERS}

A near-field wideband synthetic aperture beamformer was set up in an anechoic chamber at Naval Sea Systems Command (NAVSEA) in the
Naval Surface Warfare Center, Philadelphia, PA. A signal of $1 \mathrm{GHz}$ bandwidth with a center frequency of $2 \mathrm{GHz}$ was chosen for imaging. An HP-8510 network analyzer was used to synthesize the $1 \mathrm{GHz}$ signal, using 801 frequencies with a step size of $1.25 \mathrm{MHz}$. A conducting sphere of $12^{\prime \prime}$ diam, located at a range of $6.725 \mathrm{~m}$ and angle $0^{\circ}$, was used as the target and is shown in Fig. 5(a). Two horn antennas, both with operational bandwidths from 1.0 to $12.4 \mathrm{GHz}$, were used as transmitter and receiver. These antennas, shown in Fig. 5(b), constitute the single transmitter and single receiver subarray used to synthesize a 4-element transmit and an 8-element receive line array.

\section{A. Transmit and Receive Array Design}

Sensor placement is designed with the following physical constraints.

1) The dimension of the anechoic chamber limits the combined span of transmit and receive arrays, thereby limiting the maximum angular resolution that could be achieved.

2) The size of the antenna array element sets a minimum spacing at any time between the transmitter and the receiver.

In addition, since the transmitter and the receiver must be physically moved to different locations constituting the arrays, it is desirable for the transmit and receive array apertures to be physically non-overlapping for ease of implementation.

Based on these constraints, we designed the transmit and receive array apertures so as to achieve a sum coarray at $2 \mathrm{GHz}$ of length $15.5 \lambda_{c}$, where $\lambda_{c}$ is the wavelength corresponding to the $2 \mathrm{GHz}$ center frequency. This would give us a spatial resolution of $0.43 \mathrm{~m}$ at a range of $6.7 \mathrm{~m}$. The transmit and receive array locations are given in Table I.

The positioning system, devised to physically move the single transmitter and single receiver to the various locations of Table I, is shown in Fig. 5(c). Fig. 6 shows the plan of the anechoic chamber.

\section{B. Wideband Sum Coarray}

Fig. 7(a) shows the sum coarray $C_{S}$ corresponding to the designed transmit and receive array apertures. As discussed earlier, since the coarray locations are scaled by $1 /$ wavelength corresponding to the various frequencies of operation [3], the operation at multiple frequencies adds additional points at specific location in the sum coarray. Therefore, for wideband stepped-frequency operation, the sum coarray is the union of the dilated sum coarrays corresponding to all 801 frequencies constituting the $1 \mathrm{GHz}$ bandwidth, 


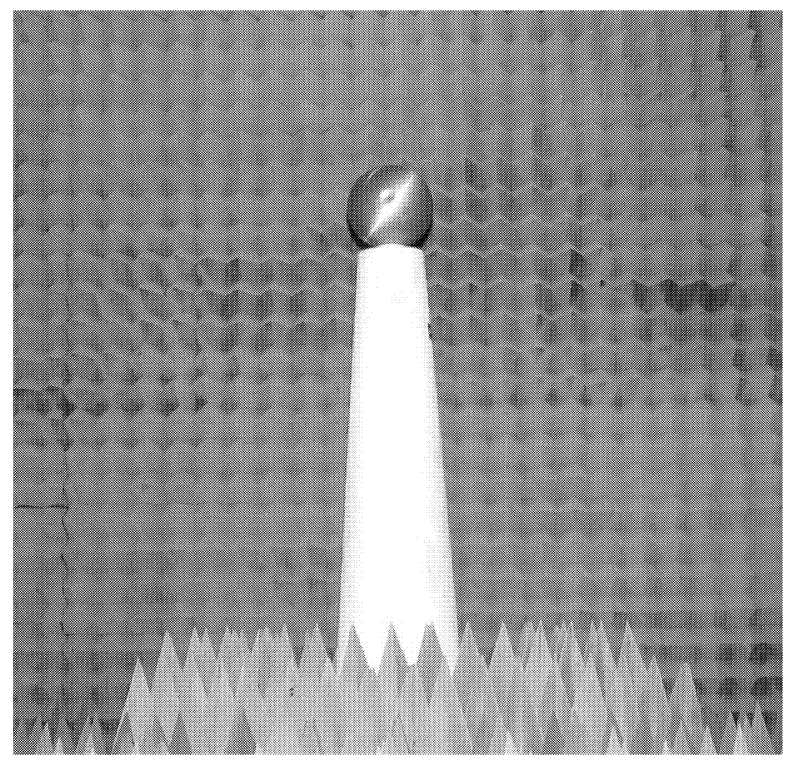

(a)

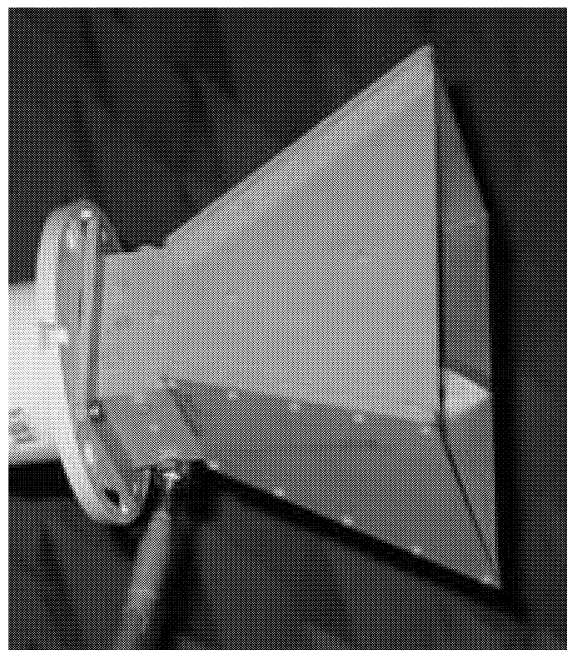

(b)

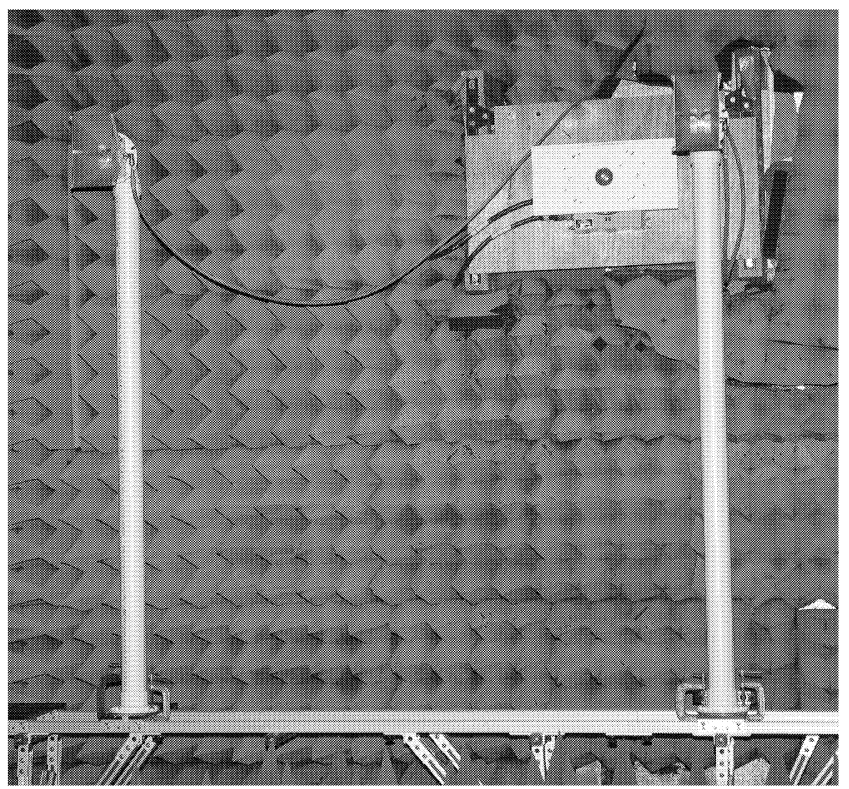

(c)

Fig. 5. (a) Conducting sphere of $12^{\prime \prime}$ diameter used as target. (b) Horn antenna. (c) Positioning system used to synthesize transmit and receive array apertures.

TABLE I

Transmit and Receive Array Locations

\begin{tabular}{ccc}
\hline \hline & $\begin{array}{c}\text { Transmitter Location } \\
(\mathrm{m})\end{array}$ & $\begin{array}{c}\text { Receiver Location } \\
(\mathrm{m})\end{array}$ \\
\hline 1 & -1.6125 & 0.450 \\
2 & -1.0125 & 0.525 \\
3 & -0.4125 & 0.600 \\
4 & 0.1875 & 0.675 \\
5 & & 0.750 \\
6 & & 0.825 \\
7 & & 0.900 \\
8 & & 0.975 \\
\hline
\end{tabular}

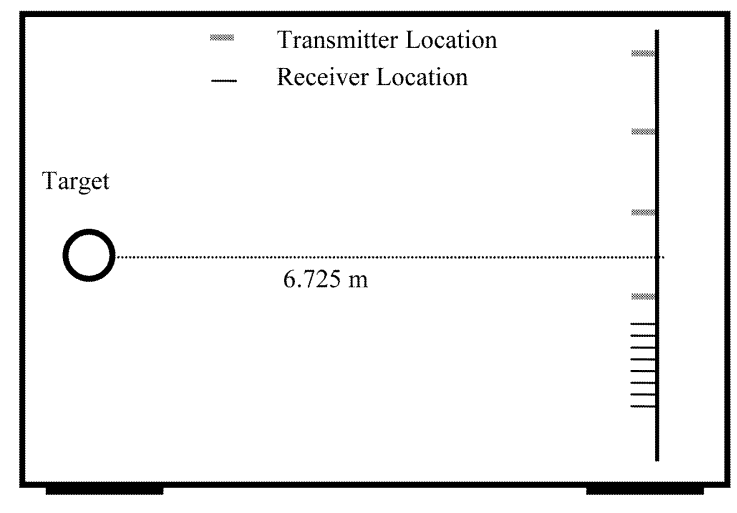

Fig. 6. Plan diagram of anechoic chamber. 
Fig. 7. (a) Sum coarray locations in meters corresponding to designed transmit and receive arrays of Table I. (b) Wideband sum coarray corresponding to $1 \mathrm{GHz}$ bandwidth.

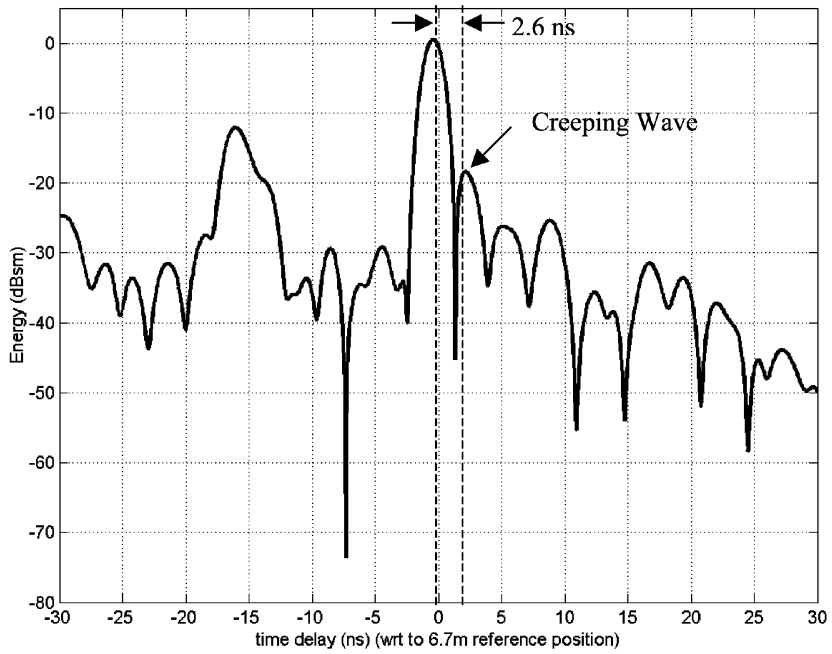

(a)

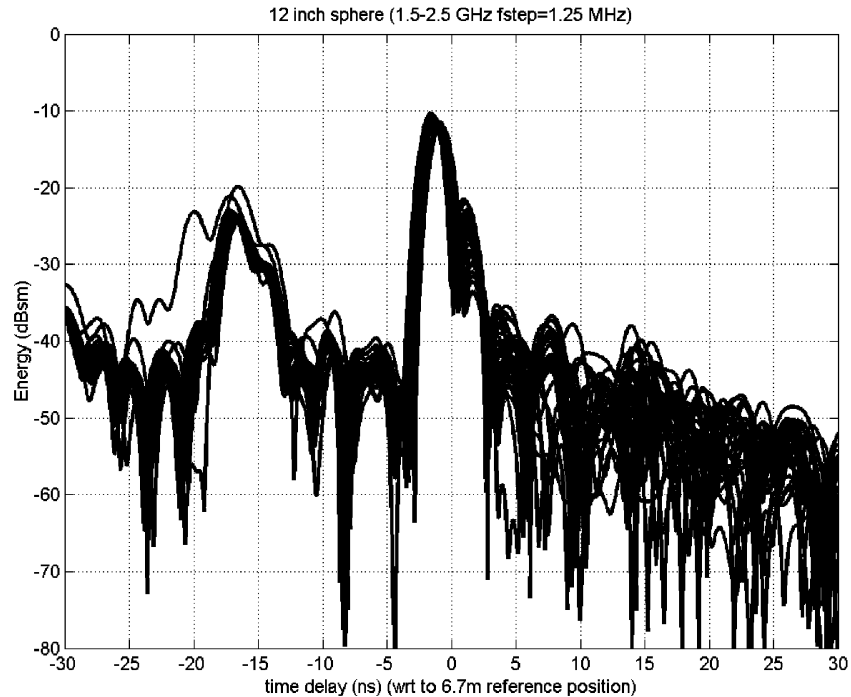

(b)

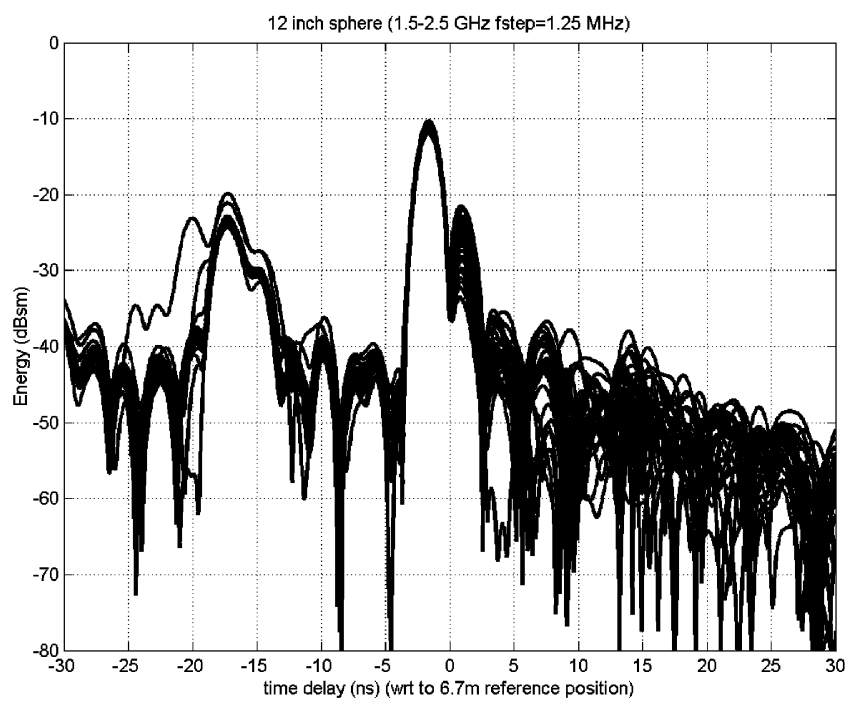

(c)

Fig. 8. (a) Synthesized time response of $12^{\prime \prime}$ sphere using 32nd transmit/receive pair (4th transmitter location and 8th receiver location). (b) Superimposed time responses of $12^{\prime \prime}$ sphere due to all 32 transmit/receive subarray pairs. (c) Superimposed time-aligned responses of $12^{\prime \prime}$ sphere for boresight.

i.e.,

$$
C_{\mathrm{WB}}=\bigcup_{k=1}^{801}\left(\lambda_{k} \cdot C_{S}\right)=\bigcup_{k=1}^{801}\left\{\left(\mathbf{z} / \lambda_{k}\right) \mid \mathbf{z} \in C_{S}\right\}
$$

where $\mathbf{z}$ is the position vector of the elements of the original coarray $C_{S}$. The coarray corresponding to $1 \mathrm{GHz}$ bandwidth operation is shown in Fig. 7(b). It is evident that the wideband operation leads to a richer sum coarray.

As our objective is to verify the working of the proposed synthetic aperture beamformer, the wideband coarray was not used for optimal sensor placement. However, once incorporated, it would allow us to achieve the present resolution using fewer transmit/receive array element locations. 


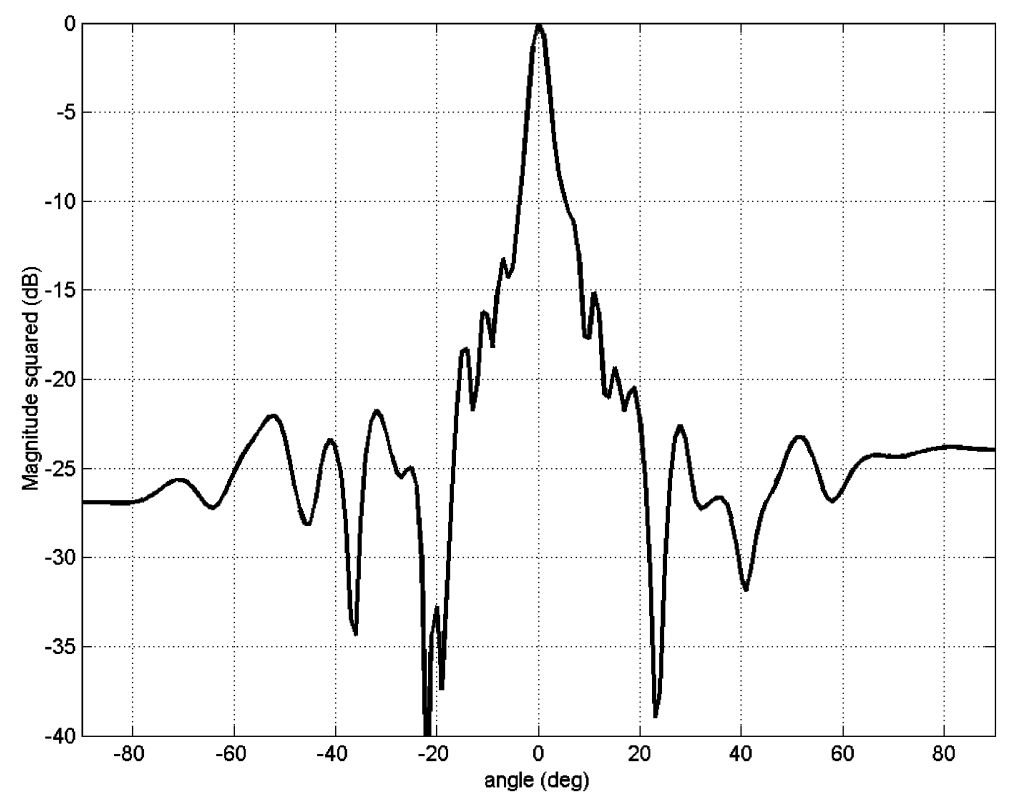

(a)

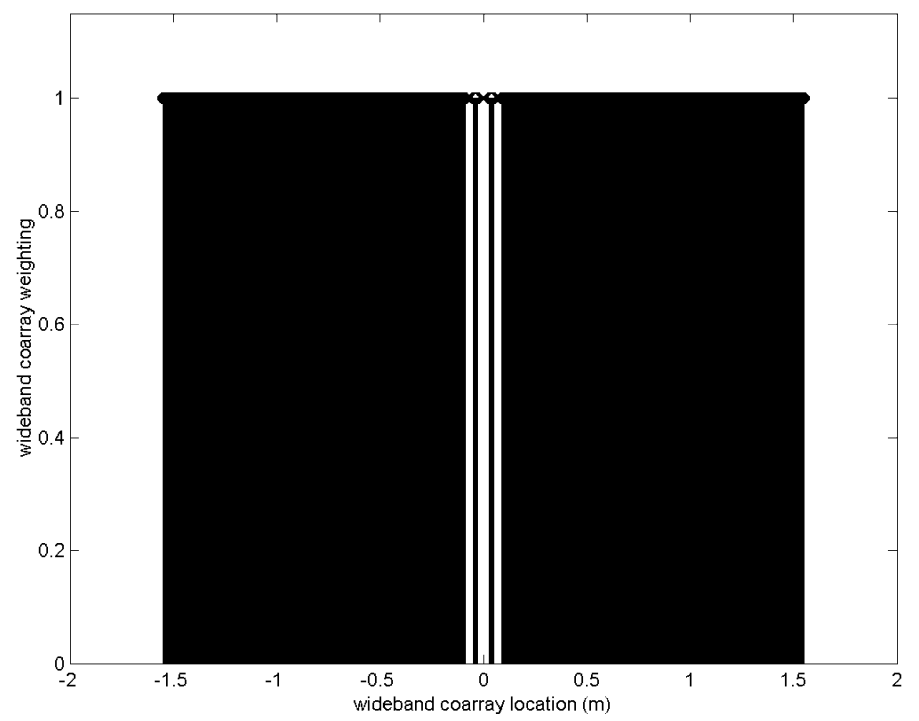

(b)

Fig. 9. (a) Normalized power pattern of array response using uniform coarray weighting and wideband sinc pulse. (b) Wideband coarray weighting resulting from application of unit weights to transmit and receive arrays.

\section{EXPERIMENTAL RESULTS}

The data measurement process for each transmit and receive location consists of the following steps.

1) Background measurement. This is a measurement made of the received amplitude and phase of all 801 monochromatic signals. The purpose of this experiment is to measure the anechoic chamber clutter characteristics, including the pedestal on which the target is placed, with only one exception, the $12^{\prime \prime}$ sphere target is not present.

2) Target measurement. This is a complex amplitude measurement of the received signal across all 801 frequencies using the same exact setting as in the first experiment, but including the $12^{\prime \prime}$ conducting sphere.

3) Background subtraction. The background data set is subtracted from the target data set for clutter reduction, although clutter due to mutual interaction between the sphere and the chamber remains, albeit at low level.

This final data set, after clutter subtraction, was used for range as well as angular processing.

In this section, we present the results of both the range and angular response of the array imaging system to the single conducting sphere, obtained using real stepped-frequency data acquired in the anechoic chamber. 


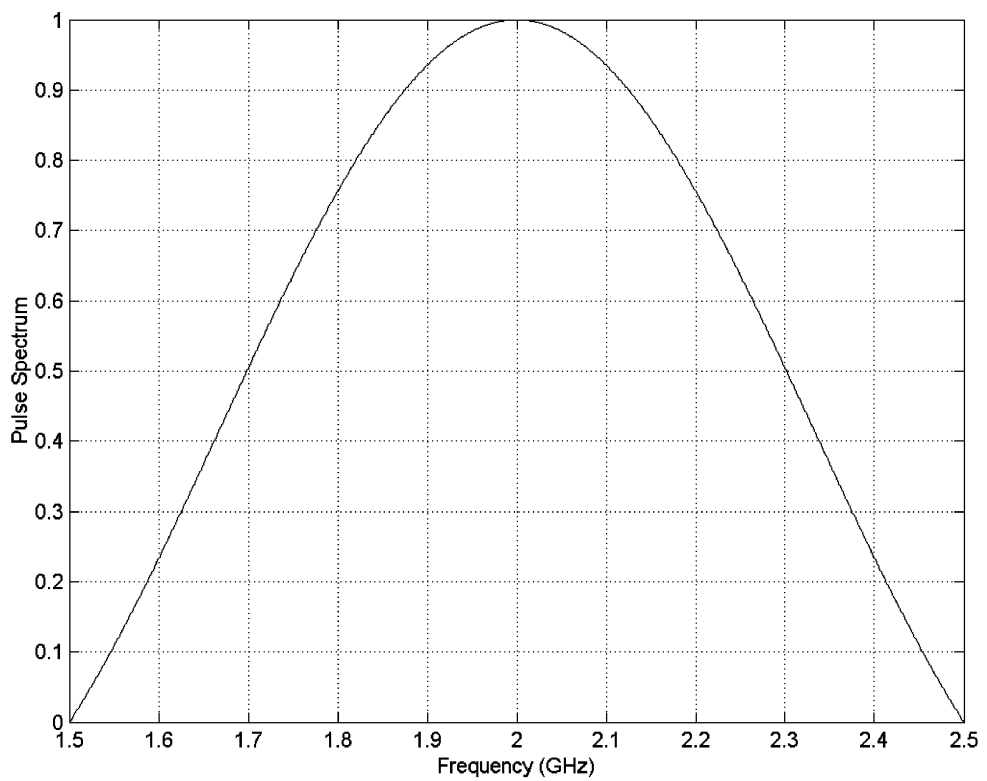

(a)

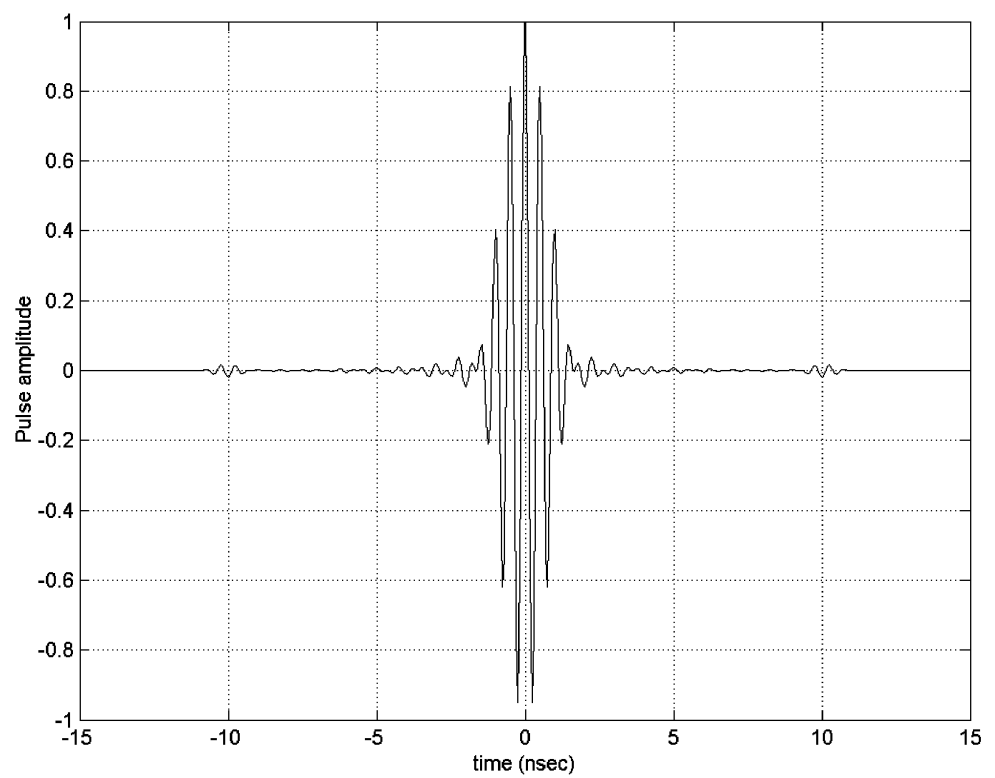

(b)

Fig. 10. (a) Tapered spectrum with bandwidth of 1 GHz. (b) Pulse with tapered spectrum of Fig. 10(a).

\section{A. Time/Range Processing}

Fig. 8(a) shows the reconstructed received wideband pulse from the stepped-frequency data for one of the transmitter/receiver pair location. The horizontal axis is time in nanoseconds and the vertical axis is the energy in $\mathrm{dBsm}(\mathrm{dB}$ relative to a square meter). The reconstruction process is an inverse Fourier transform of the background-subtracted complex amplitudes of all 801 monochromatic signals. We can see from Fig. 8(a) that although the background subtraction has been carried out, there is still clutter present due to interaction of the target with the environment. In the absence of clutter, the levels at ranges/times other than the target would have been below $-40 \mathrm{dBsm}$.

The range of the target can be determined by using the round-trip time of flight. The return immediately after the one from the sphere is due to the creeping wave phenomenon, which is the surface wave that travels around the sphere and occurs when the radius of the sphere is comparable to the wavelength of the incident signal [21]. The creeping wave return occurs after $(2+\pi)(r / c)$ seconds, where $r$ is the radius of the sphere and $c$ is the speed of light. For the sphere of $12^{\prime \prime}$ diameter, this evaluates to $2.6 \mathrm{~ns}$ and can be readily verified from Fig. 8(a). 


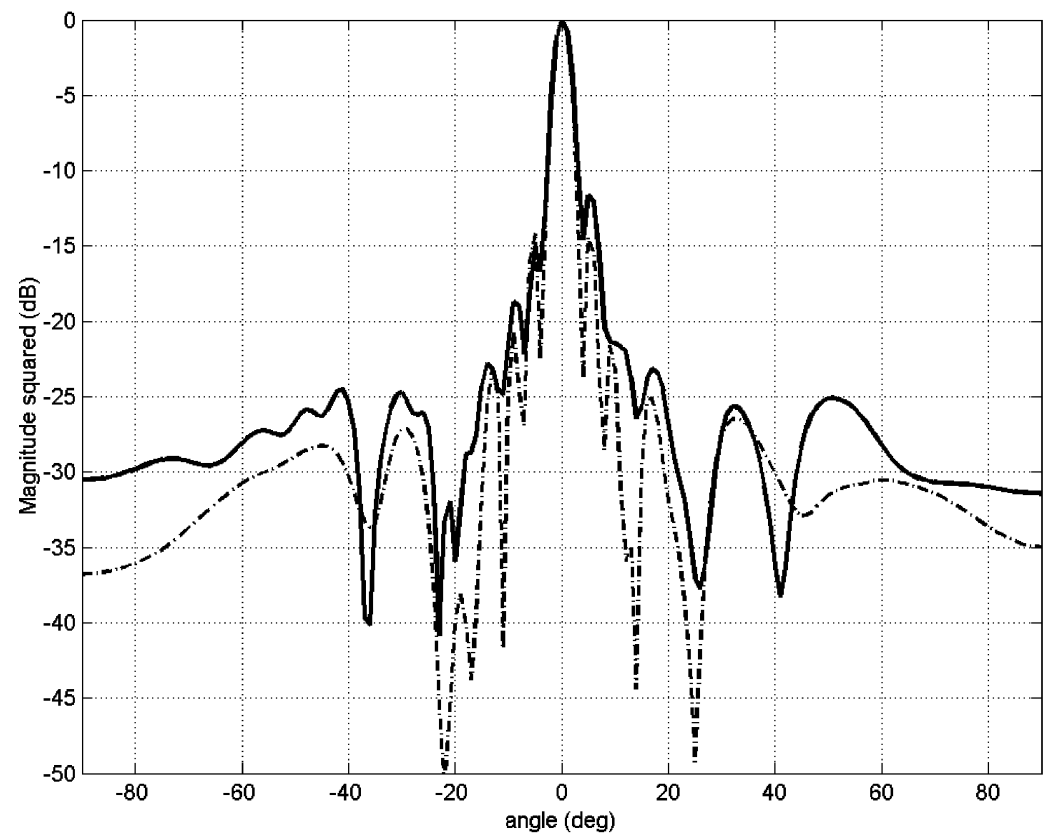

Fig. 11. Normalized power pattern of array response using coarray weighting of Fig. 9(b) and pulse of Fig. 10(b). Solid curve is obtained using experimental data. Dash-dotted curve is obtained by computer simulation.

Fig. 8(b) shows the reconstructed returns for each of the 32 transmit/receive subarray pairs. Note that the pulses in Fig. 8(b) are staggered in time, as expected, because the propagation delays encountered by the signal are different for each transmit/receive pair. Fig. 8(c) shows the same pulses after data processing for focus at the target range on boresight. We can clearly see that all the pulses have been time-aligned. As a result, they would add up to generate a large combined beamformer output, thereby accurately locating the target at an angle of $0^{\circ}$, as is shown in the following section.

\section{B. Angle/Spatial Processing}

We present array response to the spherical target computed using post-data acquisition beamforming for various pulse shapes and coarray weightings. In all the figures in this section, we plot the power pattern of the array response in $\mathrm{dB}$ units, with the maximum level normalized to $0 \mathrm{~dB}$. The range of the angular coordinate in the plots is $\left[-90^{\circ}, 90^{\circ}\right]$.

Fig. 9(a) shows the computed angular response of the array imaging system for a range of $6.725 \mathrm{~m}$ using a uniform $1 \mathrm{GHz}$ spectrum centered at $2 \mathrm{GHz}$, which corresponds to imaging using an amplitude modulated sinc pulse. Unit weights were applied to the outputs of the transmit/receive subarray pairs, which uniformly weight the narrowband sum coarray (corresponding to $2 \mathrm{GHz}$ center frequency) of Fig. 7(a). The corresponding weighting on the wideband sum coarray of Fig. 7(b) is shown in Fig. 9(b). The system clearly locates the spherical target at boresight. However, the first sidelobe is only $13 \mathrm{~dB}$ below the mainlobe level.
The angular array response was next computed using the coarray weights of Fig. 9(b) and the tapered spectrum shown in Fig. 10(a). This spectrum corresponds to the wideband pulse of Fig. 10(b), which is the filtered version of an amplitude modulated rectangular pulse consisting of four cycles of the $2 \mathrm{GHz}$ carrier. Note that this pulse is both time- and band-limited, whereas the sinc pulse used to compute the response of Fig. 9(a) was only band-limited. Fig. 11 depicts the power pattern of the array response obtained using experimental data (solid curve). We can clearly see that for the computed response, this combination of weighting and pulse spectrum leads to a reduction of $3 \mathrm{~dB}$ approximately in the sidelobe levels in the regions $\left[-90^{\circ},-10^{\circ}\right]$ and $\left[10^{\circ}, 90^{\circ}\right]$ as compared with the power pattern of Fig. 9(a). However, the first sidelobe level is still at $-13 \mathrm{~dB}$.

In Fig. 11, we also plot the array response obtained by simulating (15) using the coarray weights of Fig. 9(b) and pulse spectrum of Fig. 10(a) for comparison. We can see that the mainlobes of the computed and the simulated responses are almost identical and the sidelobe responses are also in harmony.

Using the uniform $1 \mathrm{GHz}$ spectrum and the array weights of Fig. 12(a), we calculated the angular reflectivity estimate of the single target scenario. Four sets of weights are shown in Fig. 12(a), each set applied to the 8 received returns for the specified fixed transmitter location. These weights implement the Chebyshev weighting, shown in Fig. 12(b), on the coarray of Fig. 7(a). Fig. 12(c) shows the corresponding weighting imposed on the wideband 

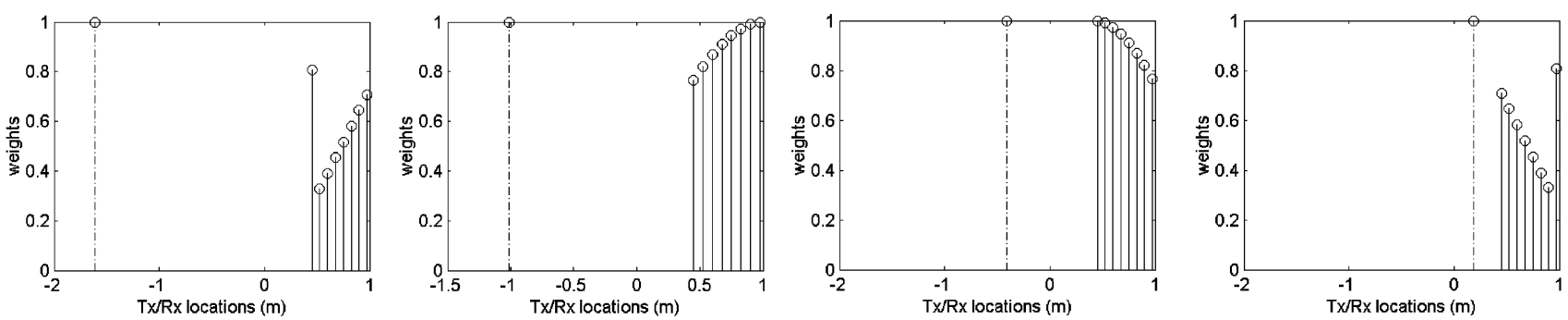

(a)

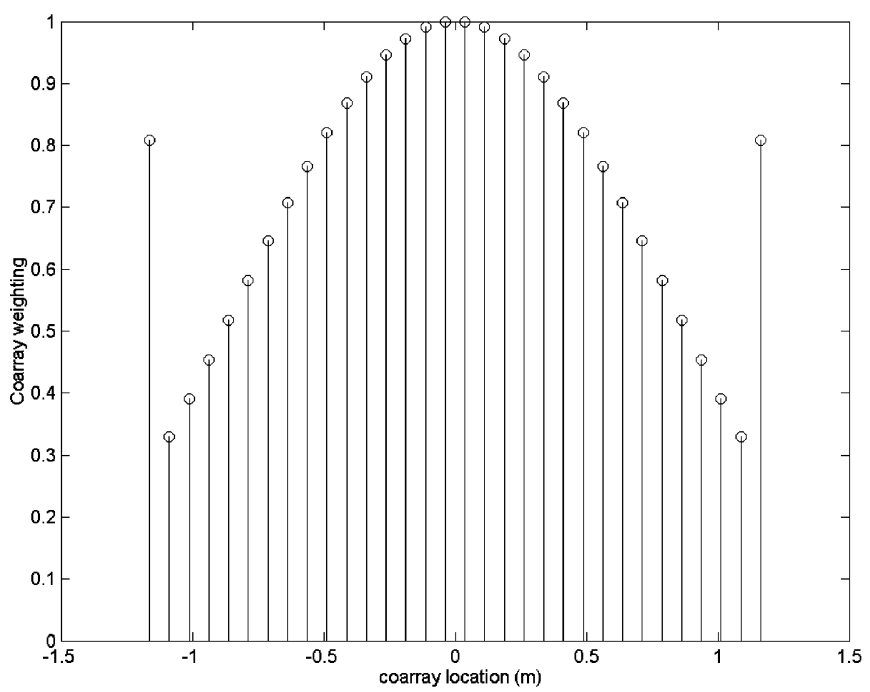

(b)

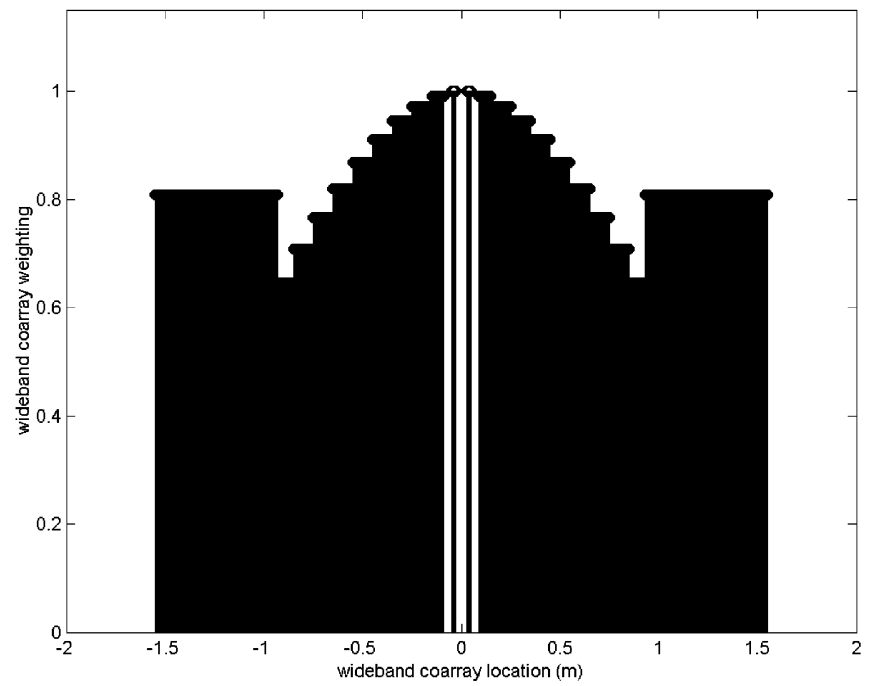

(c)

Fig. 12. (a) Four sets of weights, each applied to received signals corresponding to the 8 receive position for fixed transmitter position. (b) Corresponding Chebyshev weighting on $2 \mathrm{GHz}$ sum coarray of Fig. 7(a). (c) Corresponding weighting on wideband coarray of Fig. 7(b).

sum coarray. The angular array response is shown in Fig. 13. Again, the synthetic aperture beamformer has correctly located the target. However, it is clear that this wideband coarray weighting has achieved a reduction of about $9 \mathrm{~dB}$ in the sidelobe level between $\left[-20^{\circ}, 20^{\circ}\right]$ over that of Fig. 9(a) at the expense of a slightly wider mainlobe and sidelobe levels higher by $2 \mathrm{~dB}$ in the regions $\left[-90^{\circ},-20^{\circ}\right]$ and $\left[20^{\circ}, 90^{\circ}\right]$.
Fig. 14 depicts the power pattern of the array response (solid curve) using the tapered spectrum shown in Fig. 10(a) and the Chebyshev wideband coarray weighting of Fig. 12(c). We can clearly see that this combination of weighting and pulse spectrum leads to a reduction in the levels everywhere in the sidelobe region compared with the power patterns in Figs. 9(a) and 11. This response also has considerable 


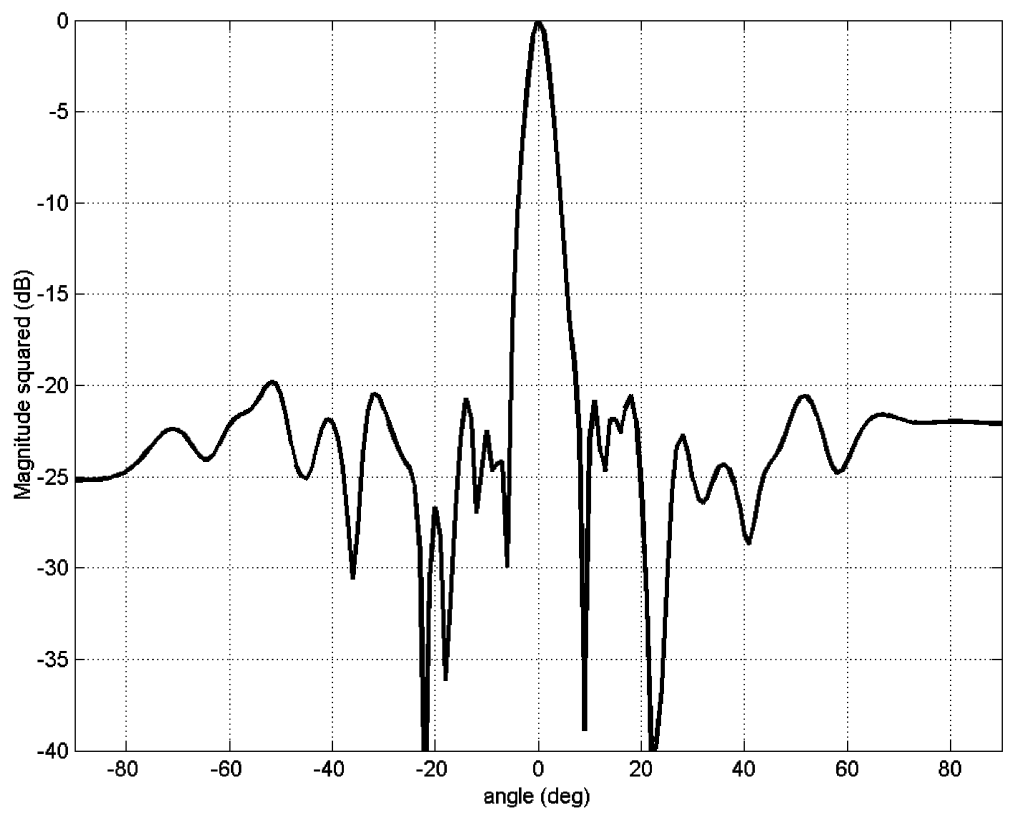

Fig. 13. Normalized power pattern of array response using coarray weighting of Fig. 12(c) and wideband sinc pulse.

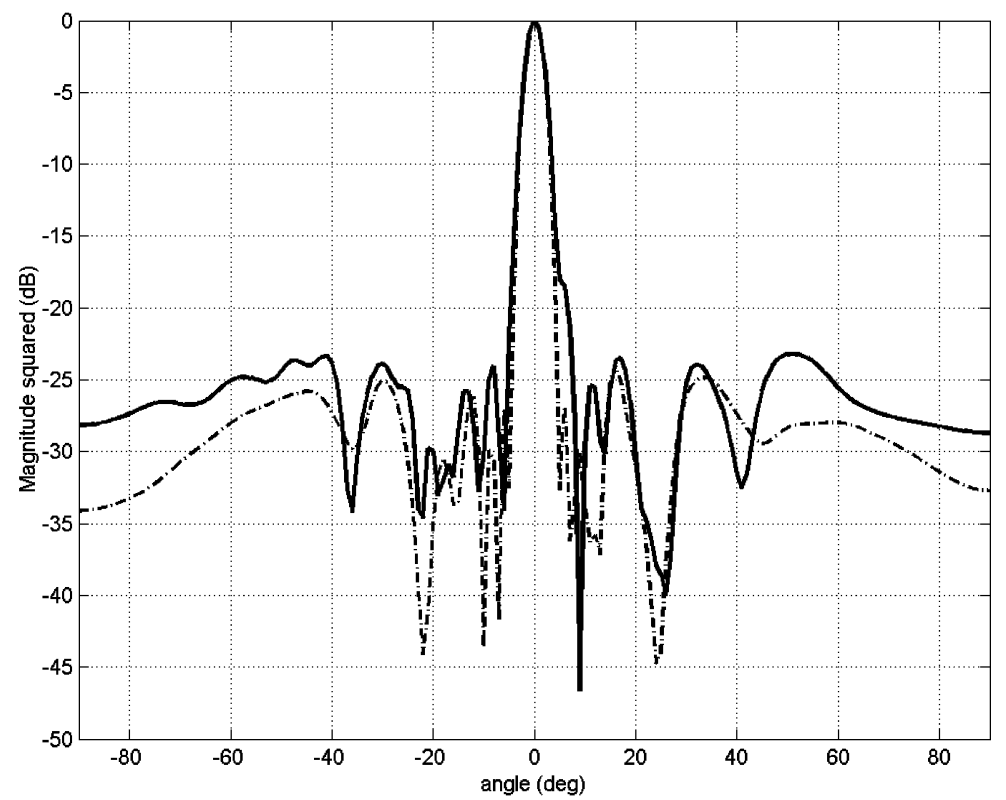

Fig. 14. Normalized array power pattern for wideband pulse of Fig. 10(b) and coarray weighting of Fig. 12(c). Solid curve is obtained using experimental data. Dash-dotted curve is obtained by computer simulation.

sidelobe level reduction in the region $\left[-40^{\circ}, 40^{\circ}\right]$, but has sidelobe levels higher by about $2 \mathrm{~dB}$ elsewhere as compared with the response in Fig. 13. We, therefore, conclude that this choice of pulse spectrum and wideband coarray weighting would give us the best result in distinguishing between two closely spaced targets when one is a weak target and the other is strong.

Fig. 14 also compares the power patterns, obtained using the experimental data, and computer simulation of (15) for the tapered spectrum of Fig. 10(a) and the coarray weighting of Fig. 12(c). Once again, we see that the mainlobes of the two responses are almost identical and the sidelobes vary in unison.

\section{CONCLUSIONS}

We have successfully designed, implemented, and demonstrated a synthetic aperture beamformer for wideband imaging of near-field scenes using stepped-frequency signal generation, coarray-based aperture synthesis, and post-data acquisition processing. We have shown that the concept of coarrays can not only be used for array aperture 
design but also can be efficiently used to weigh the received signals in order to achieve improved performance. The flexibility of the proposed design in evaluating prospective candidates for waveform design has also been shown through examples. The proposed beamformer design is particularly suitable for through-the-wall microwave imaging because of its low cost and simple implementation.

\section{ACKNOWLEDGMENTS}

The support of Naval Sea Systems Command (NAVSEA), Philadelphia, PA in allowing us to use the anechoic chamber is greatly acknowledged. Also, we would like to thank Mr. Frank Plonski, Mr. Paul Rush, Ms. Morgan Watson, and Mr. Bill Ailes of NAVSEA for their assistance in hardware setup and in data acquisition.

\section{REFERENCES}

[1] Hoctor, R. T., and Kassam, S. A. (1990)

The unifying role of the coarray in aperture synthesis for coherent and incoherent imaging.

Proceedings of the IEEE, 78, 4 (Apr. 1990), 735-752.

[2] Johnson, D. H., and Dudgeon, D. G. (1993)

Array Signal Processing-Concepts and Techniques. Englewood Cliffs, NJ: Prentice-Hall, 1993.

[3] Ahmad, F., and Kassam, S. A. (2001)

Coarray analysis of the wide-band point spread function for active array imaging.

Signal Processing, 81 (Jan. 2001), 99-115.

[4] Ahmad, F., and Kassam, S. A. (1998)

Performance analysis and array design for wide-band beamformers.

Journal of Electronic Imaging, 7, 4 (Oct. 1998), 825-838.

[5] Luthra, A. K., Kassam, S. A., and Bernardi, R. B. (1986) Body imaging using vectorial addition of acoustic reflection to achieve effect of scanning beam continuously focused in range.

U.S. Patent 4,604,697, 1986.

[6] Hoctor, R. T., and Kassam, S. A. (1996)

Array redundancy for active line arrays.

IEEE Transactions on Image Processing, 5, 7 (1996), $1179-1183$.

[7] Luthra, A. K., Kassam, S. A., and Mauchly, W. (1985) Hybrid noninvasive ultrasonic imaging system.

U.S. Patent 4,553,437, 1985.
[8] Ahmad, F., Kassam, S. A., Frazer, G. J., and Amin, M. G. (2002)

Aperture synthesis for a through-the-wall imaging system. In Proceedings of ISSPIT02, Dec. 2002.

[9] Benjamin, R., Craddock, I. J., McCutcheon, E., and

Nilavalan, R. (2002)

Through-wall imaging using real-aperture radar. In Proceedings of URSI General Assembly, Aug. 2002.

[10] Mahafza, B. R. (2000)

Radar Systems Analysis and Design Using MATLAB.

Boca Raton, FL: CRC Press, 2000.

[11] Skolnik, M. I. (1990)

Radar Handbook (2nd Ed.).

New York: McGraw Hill, 1990.

[12] Stimson, G. W. (1983)

Introduction to Airborne Radar.

Hughes Aircraft Co., El Segundo, CA, 1983.

[13] Ferris, D. D., Jr., and Currie, N. C. (1998)

A survey of current technologies for through-the wall surveillance (TWS).

Proceedings of SPIE, 3577 (Nov. 1998), 62-72.

[14] Frazier, L. M. (1996)

Radar surveillance through solid materials.

Proceedings of SPIE, 2938 (Nov. 1996), 139-146.

[15] Nag, S., Fluhler, H., and Barnes, M. (2001)

Preliminary interferometric images of moving targets obtained using a time-modulated ultra-wide band through-wall penetration radar.

In Proceedings of IEEE Radar Conference, May 2001, 64-69.

[16] Wild, N. C., Felber, F., Treadaway, M., Doft, F., Breuner, D., and Lutjens, S. (2001)

Ultrasonic through-the wall surveillance system.

Proceedings of SPIE, 4232 (2001), 167-176.

[17] Falconer, D. G., Steadman, K. N., and Watters, D. G. (1996)

Through-the wall differential radar.

Proceedings of SPIE, 2938 (Nov. 1996), 147-151.

[18] Greneker, E. F. (1998)

RADAR flashlight for through-the-wall detection of humans.

Proceedings of SPIE, 3375 (Apr. 1998), 280-285.

[19] Pillai, S. U. (1989)

Array Signal Processing.

New York: Springer-Verlag, 1989.

[20] Oppenheim, A. V., Willsky, A. S., and Young, I. T. (1983)

Signals and Systems.

Englewood Cliffs, NJ: Prentice-Hall, 1983.

[21] Born, M., and Wolf, E. (1999)

Principles of Optics: Electromagnetic Theory of

Propagation, Interference, and Diffraction of Light (7th Ed.).

Cambridge, England: Cambridge University Press, 1999.

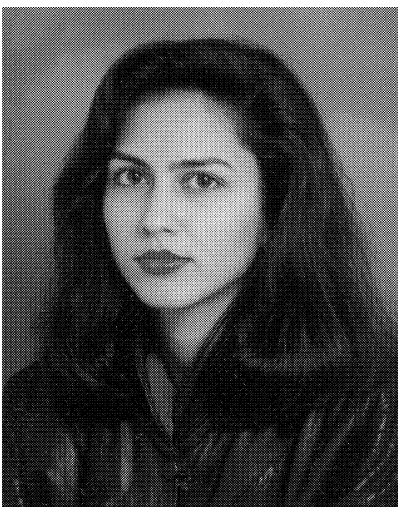

Fauzia Ahmad received her B.Sc. degree from Punjab University, Pakistan in 1990, M.Sc. degree in electronics from the Quaid-e-Azam University, Pakistan in 1993, MSEE degree in electrical engineering in 1996, and Ph.D degree in electrical engineering in 1997, both from the University of Pennsylvania, Philadelphia.

From 1998 to 2000, she was an assistant professor with the National University of Sciences and Technology, Pakistan. In 2001, she was an assistant professor at Fizaia University, Pakistan. She is currently working as a postdoctoral research fellow in the Center for Advanced Communications, Villanova University, Villanova, PA. Her research interests are in radar imaging, array signal processing, and communications. 

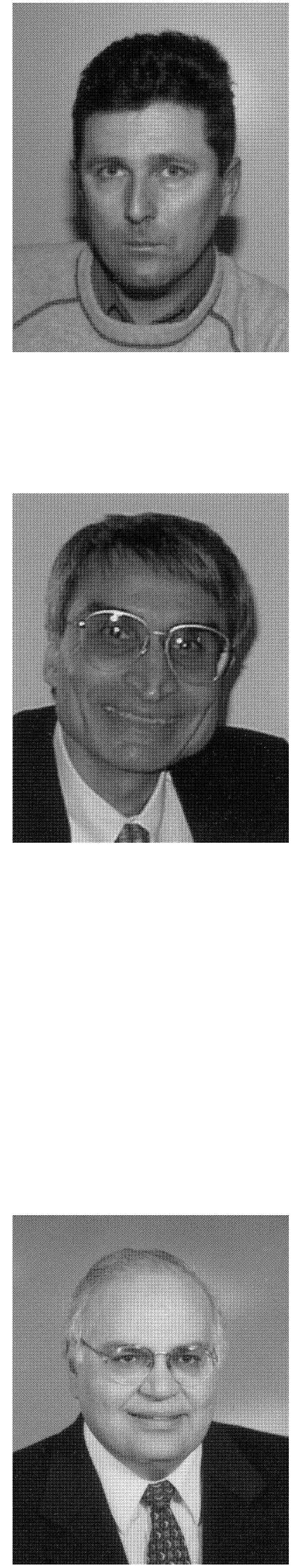

Gordon J. Frazer received the B.E. (elect.) (Hons.) degree from the University of Canterbury, New Zealand in 1982, the M.Eng.Sc degree from the University of Queensland, Brisbane in 1990 and the Ph.D degree from Queensland University of Technology, Brisbane in 1996.

From 1982 to 1988 he was a communications engineer with the Queensland Electricity Commission working on communications and control problems in electricity transmission. This included design and implementation of custom modems using digital signal processors. From 1988 until 1990 he developed a variety of custom digital signal processor applications at Mosaic Electronics Pty. Ltd. Since 1990 he has been with the Intelligence, Surveillance and Reconnaissance Division, part of the Australian Defence Science and Technology Organisation. He is presently a Principal Research Scientist and Head of Radio Frequency Projects Group. His research interests are in radar design, signal analysis and array signal processing.

Saleem A. Kassam (S'74-M'76-SM' 83-F'93) received the B.S. degree from Swarthmore College, Swarthmore, PA, in 1972, and the Ph.D. degree in electrical engineering from Princeton University, Princeton, NJ, in 1975.

Since 1975 he has been at the University of Pennsylvania, Philadelphia, where he is the Solomon and Sylvia Charp Professor of Electrical Engineering at the Department of Electrical and Systems Engineering. He is also currently director of the Graduate Program in Telecommunications and Networking. He has held visiting positions at the University of British Columbia, Vancouver, Canada, and at Princeton University. His research interests are in the areas of statistical signal processing and communication theory, with interests in nonlinear and adaptive systems, signal detection and estimation, blind equalization and source separation, fading channel modeling, image processing, and high resolution imaging systems.

Dr. Kassam is a member of Phi Beta Kappa, and has served on the Board of Governors of the IEEE Information Theory Society. He is the author of Signal Detection in Non-Gaussian Noise (Springer-Verlag, 1988).

Moeness Amin (S'82-M'83-SM'91-F'01) received his Ph.D. degree in 1984 from the University of Colorado, Boulder.

He has been on the faculty of Villanova University, Villanova, PA, since 1985, where he is now a professor in the Department of Electrical and Computer Engineering and the Director of the Center for Advanced Communications. His research includes the areas of wireless communications, time-frequency analysis, smart antennas, interference cancellation in broadband communication platforms, digitized battlefield, direction finding, and channel equalization.

Dr. Amin is the recipient of the IEEE Third Millennium Medal; distinguished lecturer of the IEEE Signal Processing Society for 2003; member of the Franklin Institute Committee of Science and Arts; recipient of the 1997 Villanova University Outstanding Faculty Research Award; recipient of the 1997 IEEE Philadelphia Section Service Award. He was the technical chair of the 2nd IEEE International Symposium on Signal Processing and Information Technology, Morocco, 2002; the general and organization chair of the IEEE Workshop on Statistical Signal and Array Processing, Pennsylvania, 2000; the general and organization Chair of the IEEE International Symposium on Time-Frequency and Time-Scale Analysis, Pennsylvania, 1994. He was an associate editor of the IEEE Transactions on Signal Processing during 1996-1998. Dr. Amin was a member of the IEEE Signal Processing Society Technical Committee on Signal Processing for Communications during 1998-2002 and was a member of the IEEE Signal Processing Society Technical Committee on Statistical Signal and Array Processing during 1995-1997. He has over 250 publications. 